\title{
Probing Expert Anticipation With the Temporal Occlusion Paradigm: Experimental Investigations of Some Methodological Issues
}

\author{
Damian Farrow, Bruce Abernethy, and Robin C. Jackson
}

Two experiments were conducted to examine whether the conclusions drawn regarding the timing of anticipatory information pick-up from temporal occlusion studies are influenced by whether (a) the viewing period is of variable or fixed duration and (b) the task is a laboratory-based one with simple responses or a natural one requiring a coupled, interceptive movement response. Skilled and novice tennis players either made pencil-and-paper predictions of service direction (Experiment 1) or attempted to hit return strokes (Experiment 2) to tennis serves while their vision was temporally occluded in either a traditional progressive mode (where more information was revealed in each subsequent occlusion condition) or a moving window mode (where the visual display was only available for a fixed duration with this window shifted to different phases of the service action). Conclusions regarding the timing of information pick-up were generally consistent across display mode and across task setting lending support to the veracity and generalisability of findings regarding perceptual expertise in existing laboratory-based progressive temporal occlusion studies.

Key Words: anticipation, occlusion paradigms, ecological validity, expert performance, tennis

One of the primary experimental approaches that have been used to examine the perceptual basis of expert anticipation in sport tasks is progressive temporal occlusion. The progressive temporal occlusion paradigm involves the editing of dynamic visual images (typically filmed from a player's perspective) in order to provide selective vision to different time periods or events within the actions of an opposing player. Multiple occlusion points are characteristically used so as to

Farrow is with Athlete and Coach Services, Australian Institute of Sport, Belconnen, ACT, 2616 Australia. Abernethy and Jackson are with the Institute of Human Performance, The University of Hong Kong, Pokfulam, Hong Kong; Abernethy is also with the School of Human Movement Studies, The University of Queensland, Brisbane QLD 4072, Australia. 
form a progressive series of viewing periods (or time windows) within the event of interest. Significant improvement in prediction accuracy across successive occlusion points is taken as evidence for information pick-up from the events contained within the viewing period bounded by these occlusion points. Prediction accuracies superior to chance levels (i.e., superior to $50 \%$ in a two-choice prediction task) are also frequently used within this paradigm to provide confirmatory evidence for significant information pick-up.

Studies using the progressive temporal occlusion paradigm (e.g., Abernethy \& Russell, 1987; Goulet, Bard, \& Fleury, 1989; Jones \& Miles, 1978; Wright, Pleasants, \& Gomez-Meza, 1990) have consistently demonstrated, across a variety of sports and a variety of different occlusion times, that not only are experts able to anticipate more effectively than novices, but they are also capable of picking up useful anticipatory information from early events in their opponent's movement pattern to which novices are not attuned. In racquet sports, for example, evidence from the temporal occlusion approach has been used to demonstrate that experts are more capable than less skilled players of picking up anticipatory information from the kinematics of events occurring early in the opponent's hitting action, such as the motion of the arm holding the racquet (Abernethy, Gill, Parks, \& Packer, 2001; Abernethy \& Russell, 1987; Buckolz, Prapavesis, \& Fairs, 1988; Goulet et al., 1989).

Despite the widespread use of the progressive temporal occlusion approach, two unresolved issues pose a threat to the validity of the conclusions that have been reached using this paradigm. The first issue relates to a potential confound in the progressive temporal occlusion paradigm between effects due to actual anticipatory information pick-up and effects due rather to intercondition variations in the length of the viewing period (and consequently the time available for visual information processing). The second issue is one of ecological validity and relates to the question of whether the film-based approaches and coupled simple response modes used in the majority of temporal occlusion studies offer a sufficiently precise simulation of the natural task demands of sport tasks to allow reliable inferences to made to the nature of perception and movement expertise as it occurs in situ. This paper seeks to examine, experimentally, the extent to which these issues may indeed compromise existing and future conclusions that are drawn from the progressive temporal occlusion paradigm.

\section{Viewing Period Duration as a Potential Confound Within Temporal Occlusion Paradigms}

A defining characteristic of the progressive temporal occlusion approach is the use of multiple occlusion conditions with systematically less of the display occluded, and more visual information available, from one occlusion condition to the next. For example, the first occlusion condition might allow vision of a tennis service action until the commencement of the ball toss while the next occlusion condition would include all this information plus additional vision of the service action, perhaps up until when the peak height of the ball toss occurs. The persistent assumption behind such an approach is that changes in performance between successive occlusion conditions must be due to the availability and pick-up of important information in the period between the two occlusions (e.g., information from the ball toss present in 
the second but not the first occlusion condition). However, it may be presumptuous to attribute a change in performance across successive occlusion conditions solely to the specific addition of new information in the display, as the later occlusion conditions not only provide more information but also an extended viewing period and hence a greater overall time in which to process visual information from the unfolding display. It is conceivable that experts and novices may differ in their capability to use, or their dependence on, this extended viewing and processing time (e.g., Adam \& Wilberg, 1992; Deary \& Mitchell, 1989). Confirmation of the prevailing assumption of active information pick-up from later occlusion conditions is required through demonstration that similar patterns of prediction accuracy improvement, as have been previously observed for instances in which the total viewing period progressively increases from the earlier to the later occlusion conditions, also occur for instances where the total viewing period is kept constant across the different occlusion conditions.

In this paper we describe experiments specifically designed to determine whether the same conclusions are reached about the advance pick-up of information to predict the direction of a tennis serve when a fixed rather than a variable duration viewing period is used with the location of the viewing period systematically moved to (un)cover different events/time periods in the opponent's action. The methodology of a variably located but fixed duration viewing period is one that has been used successfully previously in studies examining the timing of essential visual information to support catching (e.g., Sharp \& Whiting, 1974; Whiting \& Sharp, 1974). Comparing prediction accuracy under fixed and variable viewing periods (achieved respectively through moving window and traditional progressive temporal occlusion methods) provides a means of determining whether superior information pick-up actually occurs from later-occurring events in the opponent's hitting action or whether, rather, the superior performance that is characteristically seen on later occlusion conditions, is more a function of the longer viewing periods that generally exist for these conditions. Finding similar patterns of improvement in prediction accuracy across adjacent occlusion conditions under the traditional (variable viewing time) approach and the moving window (constant viewing time) approach will provide support for the current practice of assuming performance improvements across time windows occur as a direct result of active information pick-up from the later occlusion conditions. Conversely, finding evidence of prediction improvements across successive time windows under the progressive occlusion approach that are not reproduced in the moving window approach will provide support for the counter-proposition that many of the improvements in later occlusion conditions, that are currently assumed to be due to active information pick-up from these particular time periods, may be actually more a function of the extended overall viewing and processing time afforded by these conditions.

\section{Ecological Validity and Temporal Occlusion Paradigms}

To date, the majority of studies employing a temporal occlusion approach have used a two-dimensional film or video display and have required the participants to make 
only a perceptual judgment and not the usual motor response to the display. Both the display characteristics and the response mode used in these laboratory-based studies are clearly different from that typically encountered or required in the natural task and this raises issues as to the representativeness, validity, and legitimacy of laboratory-based temporal occlusion findings to perception and action as it occurs in the natural setting. This is an especially important consideration for the study of expert performance given evidence that the use of experimental conditions that do not replicate the specific perception-action couplings of the natural task may limit the extent to which expert performance can be demonstrated and consequently may provide an inaccurate insight into the nature of expert performance (Bootsma, 1989; Davids, 1988).

While the increasing use of life-size visual image projections and more sportspecific forms of responding (e.g., Helsen \& Pauwels, 1988; Paull \& Glencross, 1997; Williams, Ward, Knowles, \& Smeeton, 2002) has gone some way toward the improved realism of the testing environment, the essential uncoupling of perception and action within these approaches still remains potentially problematic. In contrast, the increasing sophistication and availability of liquid crystal occlusion spectacles (Milgram, 1987) has provided the opportunity to progress ecological validity further, to the point where it is now be possible to effectively use progressive temporal occlusion paradigms directly within the natural setting. Preliminary studies exploring this opportunity (Abernethy et al., 2001; Farrow \& Abernethy, 2003; Starkes, Edwards, Dissanayake, \& Dunn, 1995) have provided some evidence in support of the expert-novice differences found in laboratory tasks being replicable in the natural setting but further confirmatory evidence is needed. For example, the study by Farrow and Abernethy (2003) indicated that expert superiority is more evident in tasks requiring a coupled, natural response (hitting the ball) than an uncoupled, simple verbal responses (categorical prediction of stroke direction).

Our purpose in this paper is to accrue experimental evidence to directly assess the significance of the two methodological issues we have identified in relation to the progressive temporal occlusion method. We report, in total, on two experiments. The first experiment is conducted within a traditional laboratory setting and uses controlled video-based occlusions and simple verbal predictions of stroke direction to assess the extent to which variable and fixed viewing periods return comparable conclusions regarding advance information pick-up. The second experiment repeats the comparison of prediction accuracy under fixed and variable but within a natural setting in which occlusions are controlled using liquid crystal occluding spectacle technology and real movement, rather than verbal, responses are required from the participants. This second field-based experiment provides not only an additional test of the moving window-progressive temporal occlusion comparison but also makes possible, through comparison with the first experiment, examination of the second issue of concern viz., whether the expertise differences in advance information utilization that have been reported in laboratory-based temporal occlusion paradigms are indeed present when similar methodologies are applied in a natural performance setting. 


\section{Experiment 1: Method}

\section{Participants}

The participants in Experiment 1 were 11 skilled and 16 novice tennis players. The skilled participants had a mean age of 19.7 years, an average of 11.4 years of playing experience, and devoted between 20-30 hours to tennis practice each week. At the time of testing, all the skilled players were playing on the professional circuit or were members of the elite Australian Institute of Sport tennis program. The novice players, who were undergraduate students from the University of Hong Kong, had a mean age of 22.3 years $(S D=2.65)$. None of the novice players was currently playing nor had previously played competitive tennis, although all had seen tennis played and were conversant with the general requirements of the sport. All participants provided informed consent prior to undertaking the experiment.

\section{Task Design and Procedures}

The participants were shown a series of 144 video clips each depicting the service action of one of two male tennis players. The video was projected to create a near life-size image of the server and the participants' task on each trial was to predict, from the vision available, whether the ball was being served to the left or to the right of the receiving player. Responses were made by simply circling the appropriate prediction on a two-choice response sheet during the $5 \mathrm{~s}$ interval that separated each trial. A visual prompt was provided $1 \mathrm{~s}$ prior to the commencement of each trial. No auditory information was available during the task to assist the participants.

The players shown on the video were both right-handed and of standard intermediate to that of the two groups of participants. The video was filmed so as to create a viewing perspective as close as possible to that which would be available to a player on-court waiting to hit a return-of-service stroke. A large number of serves were initially filmed and from these a total of eight serves - four directed to the receiver's right and four to the left-were ultimately selected for each player for editing and inclusion in the video task proper.

Two different video task conditions were created from the selected serves in order to examine the impact of processing time on the prediction of service direction. One condition was a typical progressive temporal occlusion condition (see Abernethy \& Russell, 1987) in which visual information was progressively occluded at a number of different time periods during the server's hitting action thus providing the participants with vision of varying degrees of movement pattern and ball flight information (see Figure 1). Within this condition, the overall viewing periods for the earliest occlusions were significantly shorter in duration than those for the later occlusions. The second condition was a moving window condition, in which a fixed duration $(300 \mathrm{~ms})$ viewing period was provided at differing phases during the service action. The moving window simply shifted to a different phase of the serve on different trials while the rest of the display before and after the viewing period remained occluded (see Figure 2). In the progressive temporal occlusion condition participants could see continuous vision of the server's prepa- 


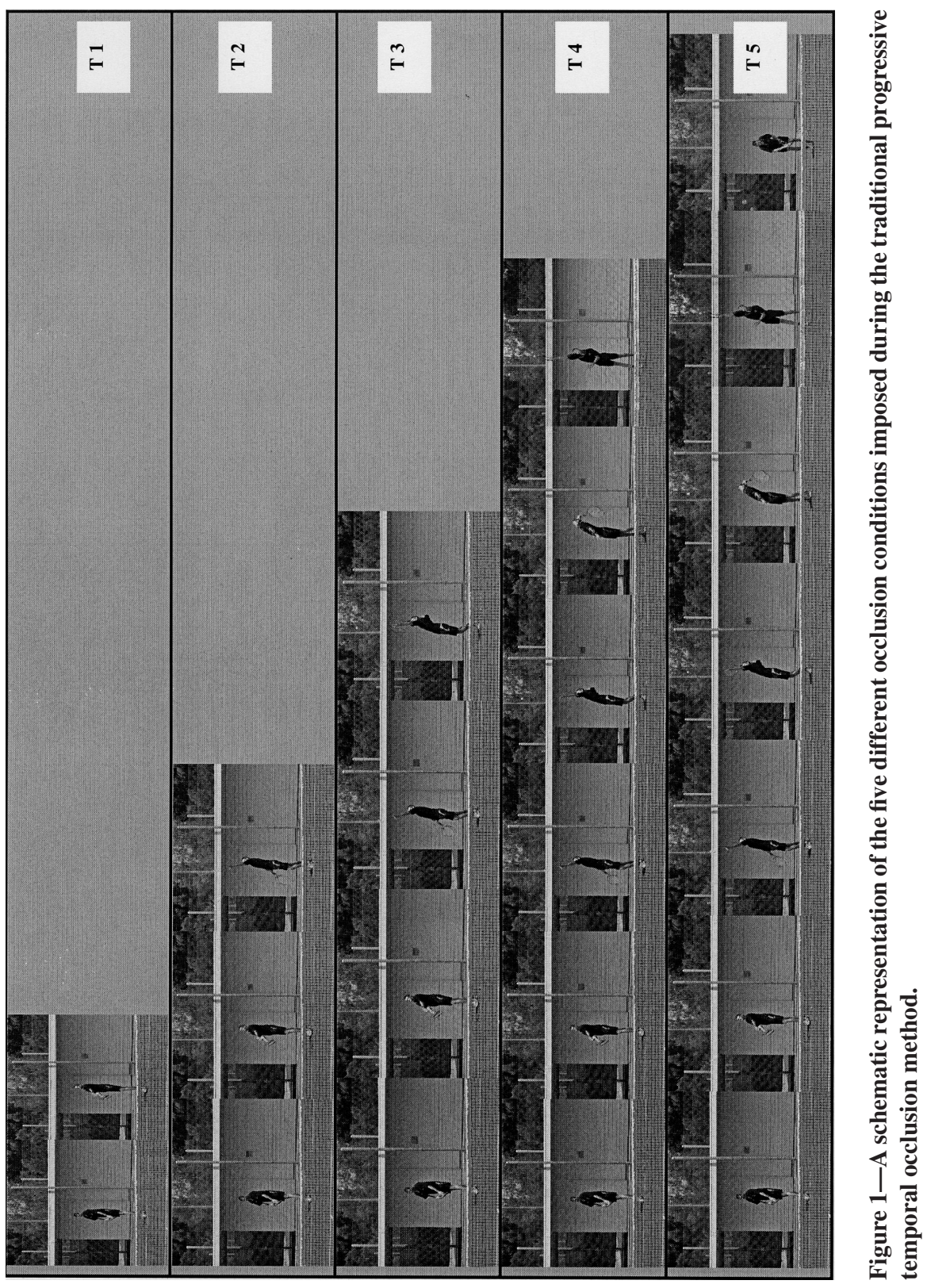




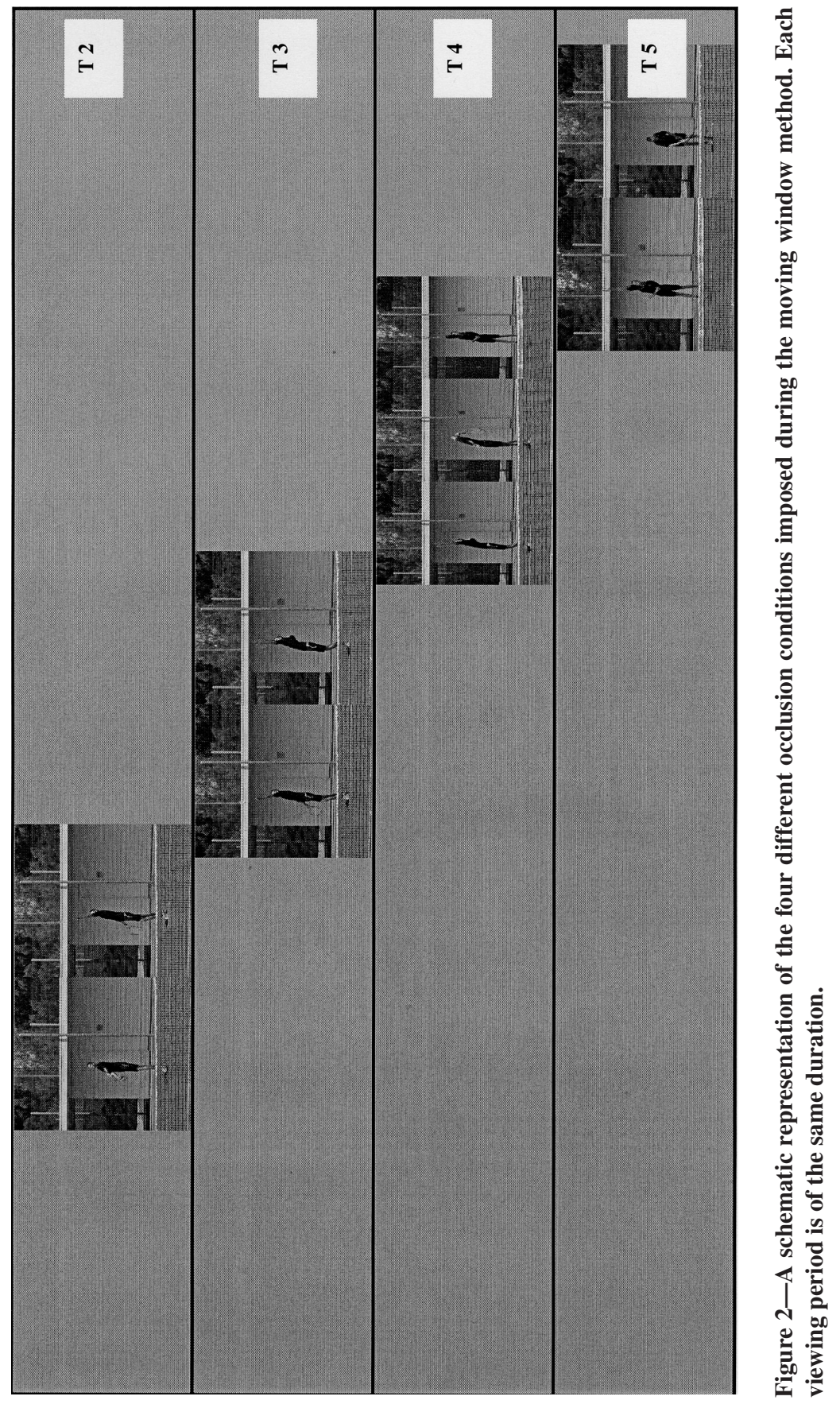


ratory actions and movement pattern up to the point of display occlusion whereas in the moving window condition vision was intermittent with vision of the server preparing to serve and vision of $300 \mathrm{~ms}$ of the service action interspersed with a period of no display information.

Four different levels of temporal occlusion were presented within the moving window condition with the $300 \mathrm{~ms}$ viewing window finishing at either $600 \mathrm{~ms}$ prior to racquet-ball contact (t2), $300 \mathrm{~ms}$ prior to contact (t3), at the point of contact (t4) or $300 \mathrm{~ms}$ after racquet-ball contact ( $\mathrm{t} 5$ ). The progressive temporal occlusion condition also included occlusions commencing at each of the display points corresponding to $\mathrm{t} 2$ - $\mathrm{t} 5$ in the moving window condition plus an additional occlusion commencing at the earlier point of $900 \mathrm{~ms}$ prior to racquet-ball contact (t1). A total of 80 trials were presented under the progressive temporal occlusion condition ( 2 servers 5 levels of temporal occlusion 8 original serves -4 in each direction) and a total of 64 trials under the moving window condition ( 2 servers 4 levels of temporal occlusion 8 original serves). Trials were blocked by server and by condition for presentation. To help combat any possible order effects across conditions of presentation, all participants completed in order the progressive temporal occlusion task for server 1 , the moving window task for server 1 , the moving window task for server 2 , and then the progressive temporal occlusion task for server 2. Fourteen practice trials were presented for each of the four server-presentation condition blocks with the total experiment taking each participant approximately $60 \mathrm{~min}$ to complete.

\section{Analysis of Data}

A single dependent measure-the percentage of correct predictions of stroke direction-was derived and subjected to a three-way (skill group display condition occlusion) ANOVA, with repeated measures on the last two factors. ${ }^{1}$ There were two levels of the skill group factor in the analysis (skilled and novice); two levels of the display condition factor (the normal, progressive occlusion condition and the moving window condition); and the occlusion factor had four levels (the occlusions $\mathrm{t} 2, \mathrm{t} 3, \mathrm{t} 4$ and $\mathrm{t} 5$ that were present for both display conditions). Greenhouse-Geisser adjustments to the degrees of freedom were applied, where necessary, to account for any deviations in sphericity within the data sets and planned contrasts were used to test more specific predictions within and across occlusion points. For the progressive temporal occlusion condition, an additional analysis was undertaken for each skill group to compare prediction performance at occlusion t1 (a level not included in the moving window condition) with that at $\mathrm{t} 2$. Paired t-tests were used, with a Bonferroni correction made to control for potential inflation of experimentwise error associated with the use of the $\mathrm{t} 2$ data in multiple analyses.

\footnotetext{
${ }^{1}$ A preliminary four-way ANOVA including server as an additional factor revealed a main effect for server, $\mathrm{F}(1,27)=11.11, \mathrm{p}<.05$, but no simple interaction with the other factors of skill, $\mathrm{F}(1,27)=$ 3.22 , $\mathrm{p}>.05$; display condition, $\mathrm{F}(1,27)=0.25$, $\mathrm{p}>.05$; or occlusion level, $\mathrm{F}(2.44,65.73)=1.73$, $\mathrm{p}$ $>.05$ nor any higher level interactions $(\mathrm{p}>.05)$. One server was therefore universally easier to predict than the other but, as this did not interact with any other factors in the analysis, the server factor was able to be legitimately eliminated from the main analysis.
} 


\section{Results and Discussion}

Figure 3 shows prediction accuracy for the skilled and novice participants as a function of the display condition (progressive temporal occlusion v. moving window) and the time of occlusion.

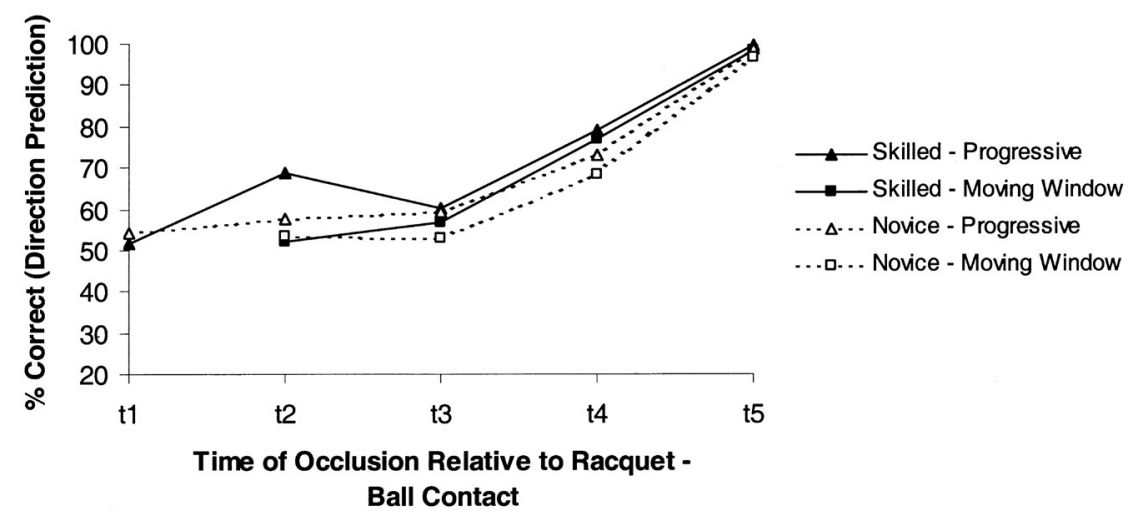

Figure 3-Prediction accuracy in Experiment 1 as a function of skill level, display condition, and time of occlusion.

Display condition significantly affected prediction accuracy, $\mathrm{F}(1,25)=9.31$, $\mathrm{p}<.05$, with superior mean prediction performance being possible under the usual progressive temporal occlusion condition $(\mathrm{M}=74.56, \mathrm{SE}=1.34)$ compared to the moving window condition $(\mathrm{M}=69.45, \mathrm{SE}=1.26)$. There was no interaction, however, between the display condition and either skill level, $\mathrm{F}(1,25)=0.18, \mathrm{p}>$ .05 or time of occlusion, $\mathrm{F}(2.20,54.97)=1.86, \mathrm{p}>.05$, nor was there a significant three-way effect, $F(2.20,54.97)=1.72, p>.05$. This indicates that while prediction collapsed across occlusion points was easier under the usual progressive temporal occlusion condition, this advantage was due to the style of presentation per se and not the specific display information available to the participants. It is most likely that the moving window condition had a residual decrement compared to the progressive condition because this condition provided perceptual disruption and intermittency of vision, whereas vision in the progressive condition was continuous and uninterrupted up to the point of occlusion.

Of greater interest was examination of the pattern of information pick-up across occlusion points. To this end, a main effect was obtained for time of occlusion, $\mathrm{F}(3,75)=147.13, \mathrm{p}<.05$, but again in the absence of significant interaction with any other factor (all $\mathrm{p}$ values $>.05$ ). Planned repeated contrasts between adjacent occlusion points revealed that prediction accuracy did not improve significantly from $\mathrm{t} 2(\mathrm{M}=58.09, \mathrm{SE}=2.19)$ to $\mathrm{t} 3(\mathrm{M}=57.39, \mathrm{SE}=1.87)$ but did, for both skill groups, from $\mathrm{t} 3$ to $\mathrm{t} 4 \mathrm{(M}=74.27, \mathrm{SE}=1.67)$ and from $\mathrm{t} 4$ to $\mathrm{t} 5 \mathrm{(M}=98.26, \mathrm{SE}=$ 0.59). In the progressive occlusion condition for which t1 data were also available, a significant mean improvement in prediction accuracy was also evident from $\mathrm{t} 1$ 
$(\mathrm{M}=52.61, \mathrm{SE}=2.45)$ to $\mathrm{t} 2(\mathrm{M}=63.28, \mathrm{SE}=2.80)$. Importantly, the absence of a significant interaction between display condition and occlusion indicated that the same pattern of information pick-up was seen regardless of whether the display was presented as a typical progressive one (with its variable viewing period durations) or as a moving window (with its constant viewing period duration). This demonstrates that the (usual) practice of drawing conclusions about information pick-up in progressive temporal occlusion paradigms through comparisons of prediction accuracy across adjacent time windows does indeed reflect information pick-up from these time windows and not rather an accrued benefit due to the increased viewing/processing time that is available in the later occlusion conditions.

While the overall mean prediction performance of the skilled participants $(\mathrm{M}=73.94, \mathrm{SE}=1.53)$ was superior to that of the novice participants $(\mathrm{M}=70.07$, $\mathrm{SE}=1.27)$, the skill factor just failed to reach statistical significance either as a main effect, $F(1,25)=3.79, p=.06$, or in interaction with time of occlusion, $F(3$, $75)=0.71, p>.55$. The failure to observe significant expertise effects is surprising but likely has two main causes. First, the occlusion times selected for inclusion in this particular study may be insufficiently fine-grained to reveal the usual expertnovice differences. Previous laboratory-based studies on racquet sports that have shown expert-novice differences in the timing of information pick-up have used multiple occlusion points in the period between $300 \mathrm{~ms}$ prior to contact and contact, for example, as this is the region in which the expert advantage is usually most evident (e.g., see Abernethy \& Russell, 1987). The present data show a clear trend for superior information pick-up by the more skilled players in the period between $\mathrm{t} 3$ and $\mathrm{t} 4$; the skilled players improve their prediction accuracy by $18.75 \%$ over this period compared to $13.67 \%$ for the novices. The use of additional occlusion periods in this region, say at either 100 or $150 \mathrm{~ms}$ intervals, may have helped reveal expertise effects that are concealed within the current design. Second, recent evidence also indicates that expertise effects are likely to be more evident in situations where a coupled movement response (such as actually hitting the ball) is required rather than a simple, uncoupled pencil-and-paper prediction of the type used in this experiment (Farrow \& Abernethy, 2003). Experiment 2 examines this contention by reproducing, as best as is possible, the experimental conditions of Experiment 1 in a natural setting in which coupled movement responses are required and the progressive and moving window display conditions are achieved by using liquid crystal goggles rather than video manipulation.

\section{Experiment 2: Method}

\section{Participants}

A total of 29 participants, 15 skilled and 14 novice tennis players, participated voluntarily in this study. The expert players consisted of 10 males and 5 females and had an average age of 17.6 years. They were members of either the elite Australian Institute of Sport tennis program, Queensland Academy of Sport tennis program, or were open aged nationally ranked competitors. This group had played tennis for an average of 10.2 years. The novice group consisted of 9 males and 5 
females with an average age of 19.8 years. They were drawn from the undergraduate student population of the University of Queensland and had played tennis for an average of 5.8 years. None of the participants in this experiment had participated in Experiment 1.

\section{Apparatus}

Testing in this experiment was conducted on a regulation Rebound Ace ${ }^{\mathrm{TM}}$ outdoor tennis court. Participants wore a pair of PLATO liquid crystal occlusion spectacles (Milgram, 1987) that were connected to a UHF receiver worn in a carry pack strapped to the player's back at waist level. The spectacles rapidly occluded the participant's vision (within a $3 \mathrm{~ms}$ period) by alteration of the state of the spectacle lenses from clear to opaque. Activation of the occluding spectacles was achieved via a computer/UHF transmitter interface unit controlled manually by an experimenter who was positioned at the back of the tennis court behind the receiver. The radio frequency emitted from the transmitter unit simultaneously triggered the closure of the spectacles and illumination of a light emitting diode (LED) located within the field of view of a video camera. The camera, operating at $200 \mathrm{~Hz}$, was positioned behind the receiver and was able to provide a synchronized record of the serve-return scenario. This camera also provided a video record of the movement direction of the receiver and service direction and location. The number of frames between the illumination of the LED and racquet-ball contact enabled the time of occlusion on each trial to be retrospectively determined to within $10 \mathrm{~ms}$ for each trial. Participants wore industrial strength earmuffs to negate any auditory information that may have been used as source of anticipatory information.

\section{Procedures}

All participants were required to attempt to return, to the best of their ability, tennis serves hit by two righted-handed, male servers, representative of an intermediate level of tennis skill and unfamiliar to the participants. These servers were different, but of comparable standard, to those used in Experiment 1. After a 5 min warm up session to familiarize themselves with the servers, participants were fitted with the occlusion spectacles and earmuffs and were given 10 pre-test practice trials. The purpose of these pre-test trials was to familiarize the participants with the task requirements. Participants commenced their return of serve from the intersection of the baseline and singles sideline, a position corresponding with the typical receiver's position in tennis.

As in Experiment 1, trials were presented under two different methods of display presentation (progressive temporal occlusion and moving window) and with occlusion levels comparable to those used in the earlier experiment. Five levels of occlusion were presented under the progressive temporal occlusion condition (cf. Figure 1). In condition t1, the display was occluded at or before $900 \mathrm{~ms}$ prior to racquet-ball contact at a point corresponding with the commencement of the ball toss. In condition t2, occlusion occurred at or before $600 \mathrm{~ms}$ prior to racquet-ball contact at the point where the ball toss has nearly reached its zenith. In addition to 
the information visible at $\mathrm{t} 1, \mathrm{t} 2$ also provided vision of the upward movement of the ball toss and the racquet's movement into a "Y" position with the ball toss hand. Condition $\mathrm{t} 3$ occluded the display when the racquet was at the top of the back-swing and the ball toss hovering at its zenith at a time at or before $300 \mathrm{~ms}$ prior to contact. This condition presented, in addition to vision of the information available in $\mathrm{t} 2$, vision of the server's action including the movement of the racquet head toward the start of the back-swing. Condition 44 occluded the display around the point of racquet-ball contact and consequently presented vision, in addition to that included in $\mathrm{t} 3$, of the back-swing into the "back-scratch" position, the acceleration or throwing of the racquet head up to the ball, and any final downward movement of the ball toss. Condition 5 occluded the display at a point after contact permitting vision of the server's follow-through motion plus post-contact movement of the ball until it reached the vicinity of the net. In the moving window display condition, there were four levels of temporal occlusion. Vision in condition $\mathrm{t} 2$ concluded in the period from 900-600 ms prior to contact; in condition $\mathrm{t} 3$, in the period from $600-300 \mathrm{~ms}$ prior to contact; in condition $\mathrm{t} 4$, in the period from $300 \mathrm{~ms}$ prior to contact to the point of racquet-ball contact; and in condition t5, within the first $\sim 300 \mathrm{~ms}$ after contact. Given the difficulty in the field setting in achieving precise control of the viewing onset and offset in relation to events within the service action, the viewing period duration was uniformly extended from the $300 \mathrm{~ms}$ used in the laboratory task to $500 \mathrm{~ms}$ in this experiment.

Irrespective of the occlusion methodology used, all trials commenced with introductory vision of service preparation that included the server walking up to the baseline, assuming the service stance and bouncing the ball. This initial vision was provided to ensure that all participants had an adequate orientation to each trial and were therefore primed ready to respond. Because of the difficulty in obtaining precise control of the timing of occlusion in the natural setting, it was necessary for the participants to undertake a large number of trials to ensure adequate data sampling was obtained in each occlusion condition. Consequently, all participants received a minimum of 156 serves in their testing session (60 trials under the progressive occlusion condition and 96 under the moving window occlusion condition ${ }^{2}$ ), with each of these serves hit at speeds of approximately $80-100 \mathrm{~km} / \mathrm{hr}$.

The servers followed a predetermined random schedule, identical for each participant, in order to distribute the serves as equally as possible to the left and right sides of the service box. Serves that were hit into the middle of the service box were eliminated from analysis while a trial creating a service fault or "let" was repeated only for serves under condition $\mathrm{t} 5$ where there was to be ball flight present in the trial. Each server would complete all required serves for each occlusion method before the next server commenced his trial block of the same occlusion method. The same procedure was then followed for the other occlusion method. The order of presentation of occlusion methods and servers was counterbalanced across participants.

\footnotetext{
${ }^{2} \mathrm{~A}$ larger number of trials was completed for the moving window condition in order to collect sufficient data reflective of the specific service phases displayed in the progressive occlusion approach.
} 
Analysis of Data

Participants' return of serve responses were defined by the final direction (left or right) that they moved their body in an attempt to intercept the oncoming serve. From this it was possible to calculate the percentage of successful responses for each time window-a measure that could be compared directly between occlusion conditions and methods and with the findings for Experiment 1 and other temporal occlusion studies.

Because manual triggering of the liquid crystal spectacles does not permit precise trial-to-trial control of occlusion onset, post-hoc video inspection was used to (a) determine which trials were suitable for inclusion in the analysis proper and (b) sort these trials into the appropriate level of occlusion. This post-hoc data sorting resulted in a varying number of eligible trials/data points for each participant and skill group in each display condition and occlusion level (see Table 1). As a result of this process only nine experts and seven novices had sufficient data in both display conditions and all occlusion windows to be considered in the main analyses.

The resultant data were then analyzed following the same basic approach as used in Experiment 1. The principal analysis was again a 224 (skill group display condition occlusion) repeated measures ANOVA with Greenhouse-Geisser adjustments for sphericity. Paired t-tests with Bonferroni adjustment were applied to compare the prediction accuracy under occlusion $\mathrm{t} 1$ in the progressive occlusion display condition with that under occlusion $\mathrm{t} 2$ in the same display condition. Additionally, all mean data points for each skill group were also compared against $50 \%$ to determine if the prediction performance differed reliably from that which would have arisen simply from guessing. (In a two-choice prediction task, a mean prediction level of approximately 50\% would be expected if a participant was simply guessing.)

Table 1 Number of Data Points Collected for Each Occlusion Level, Skill Group and Display Condition in Experiment 2

\begin{tabular}{lccccc}
\hline & \multicolumn{5}{c}{ Occlusion level } \\
\cline { 2 - 5 } Display condition & $\mathrm{t} 1$ & $\mathrm{t} 2$ & $\mathrm{t} 3$ & $\mathrm{t} 4$ & $\mathrm{t} 5$ \\
\hline Progressive occlusion & & & & & \\
Expert & 116 & 201 & 252 & 239 & 67 \\
Novice & 136 & 191 & 193 & 232 & 51 \\
Moving window & & & & & \\
Expert & -- & 122 & 319 & 515 & 243 \\
Novice & -- & 157 & 293 & 378 & 225 \\
\hline
\end{tabular}




\section{Results and Discussion}

Figure 4 shows prediction accuracy for the skilled and novice participants in this experiment as a function of the display condition (progressive temporal occlusion v. moving window) and the time of occlusion.

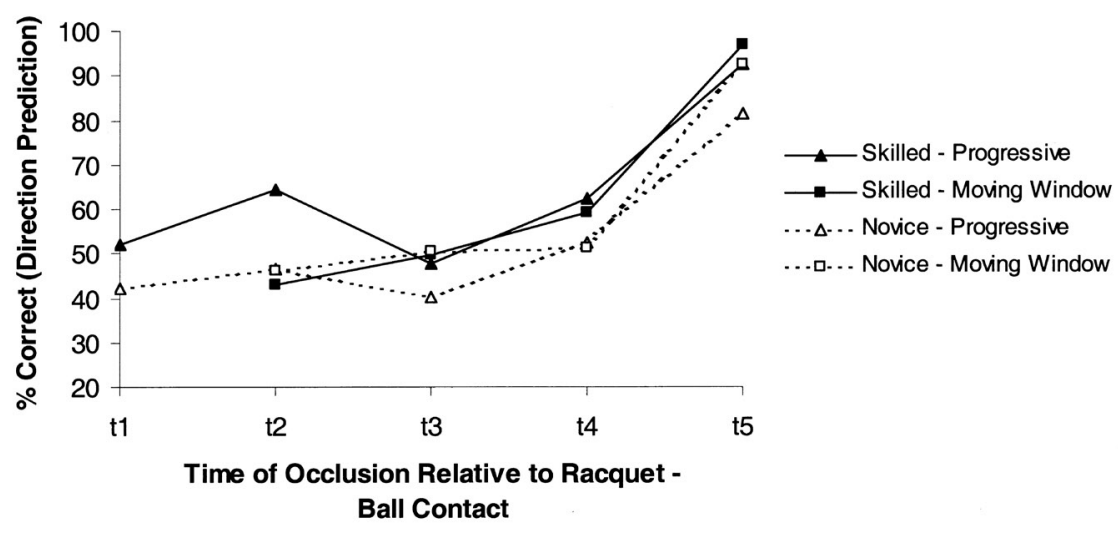

Figure 4-Prediction accuracy in Experiment 2 as a function of skill level, display condition, and time of occlusion.

In contrast to the situation in Experiment 1, there was no significant main effect for display condition in the field experiment, $F(1,14)=0.00, p>.05$, with overall levels of prediction accuracy for the progressive temporal occlusion condition $(\mathrm{M}=61.69, \mathrm{SE}=2.29)$ and the moving window condition $(\mathrm{M}=61.79, \mathrm{SE}=$ $1.56)$ being indistinguishable. A main effect was obtained for time of occlusion, $\mathrm{F}(3,42)=69.34, \mathrm{p}<.05$, due to significant increases in overall prediction accuracy from $\mathrm{t} 3(\mathrm{M}=48.06, \mathrm{SE}=2.84)$ to $\mathrm{t} 4(\mathrm{M}=58.30, \mathrm{SE}=2.03)$ and, in turn, from $\mathrm{t} 4$ to $t 5(\mathrm{M}=90.20, \mathrm{SE}=1.72)$, but this was superseded by a significant interaction between these two factors, $\mathrm{F}(3,42)=4.34, \mathrm{p}<.05$. Mean prediction accuracy progressively increased across each adjacent occlusion point for the moving window condition (means of 44.44, 51.54, 57.64, and 93.53 for occlusions $\mathrm{t} 2$, t3, $\mathrm{t} 4$ and $\mathrm{t} 5$, respectively), whereas for the progressive display condition, prediction accuracy decreased from $\mathrm{t} 2(\mathrm{M}=56.36, \mathrm{SE}=3.79)$ to $\mathrm{t} 3(\mathrm{M}=44.58, \mathrm{SE}=4.22)$ before increasing thereafter with means of 58.95 and 86.86 for occlusions t 4 and $\mathrm{t} 5$, respectively.

In this particular experiment, there was no main effect for skill level, $\mathrm{F}(1$, $14)=1.58, \mathrm{p}>.05$, although the overall prediction performance for the skilled participants $(\mathrm{M}=63.58, \mathrm{SE}=1.94)$ was higher than that for the novice participants $(\mathrm{M}=59.90, \mathrm{SE}=2.20)$. Skill, however, interacted significantly with the display condition at a simple level, $\mathrm{F}(1,14)=6.20, \mathrm{p}<.05$, with the skilled participants' best overall prediction performance being achieved under the progressive occlusion condition ( $\mathrm{M}=66.77 \mathrm{cf}$. 60.39), whereas the converse was true for the novices ( $\mathrm{M}$ 
$=56.61 \mathrm{cf}$. 63.18), although these effects were also superseded by a significant higher-order three-way interaction between skill, display condition and occlusion level, $\mathrm{F}(2.13,37.85)=3.97, \mathrm{p}>.05$.

For the skilled participants, prediction accuracy changes for the two different display conditions were comparable across the occlusion conditions $\mathrm{t} 3$ - $\mathrm{t} 4$ and $\mathrm{t} 4 \mathrm{-t} 5$ but not for the $\mathrm{t} 2-\mathrm{t} 3$ window (see Figure 4). From t2-t3, prediction accuracy for the skilled players actually deteriorated under the progressive occlusion condition, whereas it improved under the moving window condition. These effects appear to be the result of both an unexpectedly high prediction accuracy at 2 for the experts under the progressive display condition and an unexpectedly low (and poorer than chance) prediction accuracy at the same occlusion level under the moving window condition. Controlling the viewing period duration (through the moving window condition) therefore confirmed that the conclusions that would be drawn from the usual progressive occlusion paradigm regarding information pick-up by experts from $\mathrm{t} 3-\mathrm{t} 4$ and $\mathrm{t} 4-\mathrm{t} 5$ were reasonable ones but cast some doubt on the conclusions that might be drawn from the earlier $\mathrm{t} 2$-t 3 period.

For the novice participants, the patterns of prediction accuracy change across the adjacent occlusion windows were the same for the two display conditions for $\mathrm{t} 4-\mathrm{t} 5$ but different at $\mathrm{t} 2-\mathrm{t} 3$ and $\mathrm{t} 3-\mathrm{t} 4$. The differences arose primarily from a lower than expected (and lower than chance level) response at $\mathrm{t} 3$ under the progressive condition. As none of the data points for the novices prior to t5 were significantly superior to chance/guessing levels (of 50\%), it is reasonable to assume that no information pick-up of value in predicting service direction occurred under either display condition from $\mathrm{t} 1-\mathrm{t} 2$, from $\mathrm{t} 2-\mathrm{t} 3$, or from $\mathrm{t} 3-\mathrm{t} 4$ for the novices. In that respect, the conclusions regarding information pick-up that would be derived from the traditional progressive occlusion method with its variable duration viewing periods were not different from that which could be derived from the moving window condition (with its constant viewing period duration).

\section{General Discussion}

The first major purpose of this paper was to ascertain if viewing period duration is a confound that may affect the validity of conclusions about information pick-up that have been made to date from progressive temporal occlusion paradigms. The critical test in this regard was whether the same conclusions were reached regarding information pick-up from conditions in which the viewing period was continually extended with later occlusion levels (the typical progressive occlusion condition; see Figure 1) or the viewing period was held constant (through the provision of a moving window of vision; see Figure 2). In Experiment 1, where the viewing period duration and the onset of occlusion relative to events in the service action were able to be precisely controlled, the same essential conclusions regarding information pick-up were returned from both the progressive occlusion (variable viewing period duration) and the moving window (constant viewing period duration) display conditions. Under both display conditions, the consistent statistical conclusion was that significant prediction improvement for both the skilled and novice groups of participants occurred in the $300 \mathrm{~ms}$ time periods immediately prior to (t3-t4) and immediately following (t4-t5) the time of racquet-ball contact by the server but not in any other time window (see Figure 3). 
In Experiment 2, real tennis strokes were required to strike real tennis serves, improving enormously the ecological validity of the task but necessarily with some reduction in the precision of control that was achievable over the timing of the occlusions. In this second field-based experiment, quite consistent conclusions were again reached from the progressive and the moving window display conditions. Both methods of display control revealed significant pick-up of information for both the skilled and the novice participants in the post-contact (t4-t5) period and significant pick-up of information for the skilled participants in the time window immediately prior to contact ( $\mathrm{t} 3$ - $\mathrm{t} 4$; Figure 4 ). For the novices, there was no evidence of information pick-up at any earlier time periods under either display condition with all levels of prediction accuracy for $\mathrm{t} 1, \mathrm{t} 2$, and $\mathrm{t} 3$ being no better than the $50 \%$ guessing level (or in the case of $\mathrm{t} 3$, under the progressive condition being actually significantly poorer than chance). For the skilled group, prediction performance with progressive occlusion $600 \mathrm{~ms}$ prior to contact (t2) was significantly superior to guessing levels; however, this better-than-chance level of prediction was not evident for the progressive occlusion conditions either immediately before or after $\mathrm{t} 2$ ( $\mathrm{t} 1$ and $\mathrm{t} 3$ ) nor was it true for the comparable moving window occlusions. It is this particular data point ( $\mathrm{t} 2$ under the progressive display occlusion for the skilled participants) that appears largely responsible for the significant three-way skill display condition occlusion interaction that was evident in Experiment 2.

The balance of evidence from both Experiments 1 and 2 is that similar conclusions regarding information pick-up from the display are reached regardless of whether a variable or a fixed viewing period duration is presented within the occlusion paradigm. This overall finding lends support to the interpretations that have been made to date in the literature from studies using the progressive temporal occlusion approach and supports the contention that the usual progressive occlusion method does in fact provide a veridical measure of information pick-up and not simply the cumulative benefits of the additional viewing and processing time that exist within the later occlusion conditions. For both skilled and novice players, superior information pick-up occurs from later sections of the opponent's service action even when the total time available for viewing of the service action is kept constant.

In the laboratory setting of Experiment 1, the overall mean prediction performance was significantly superior under the progressive condition compared to the moving window condition-although, importantly, this did not interact with the time of occlusion and hence did not influence the overall pattern of prediction improvement/information pick-up. The suppressed performance under the moving window condition is likely due to the intermittency of vision that occurs under this condition but not under the progressive condition, although further work is needed to ascertain why this effect is not evident, or at least not uniformly so, in the field setting of Experiment 2. One possibility is that there are richer sources of supplementary stimulation available within the natural setting to help "fill in" the perceptual gap created by the intermittent occlusion to vision in the moving window condition; another is that the visual change from image to occlusion is somehow fundamentally different when occlusion is provided at the level of the stimulus (as in Experiment 1) compared to at the level of the eyes (as is achieved through the goggles technology). Studies of other motor skills such as catching (e.g., 
Bennett, Ashford, \& Elliott, 2003; Lyons, Fontaine, \& Elliott, 1997), prehension (e.g., Bennett, Elliott, Weeks, \& Keil, 2003; Elliott, Chua, \& Pollock, 1994), and balance (e.g., Robertson, Collins, Elliott, \& Starkes, 1994) have also indicated that the relative effects of visual intermittency are sensitive to task conditions such as the degree of intermittency and the information available between the intermittent samples.

The second major purpose of this paper was to examine the extent to which laboratory-based temporal occlusion studies provide conclusions regarding information pick-up and the nature of expertise effects, which are reflective of anticipatory skill as it occurs in the natural setting where the sensory information is richer, the time constraints are more restrictive, and the concurrent demand exists to couple perception with complex, interceptive action. While the participants and the servers used in the two experiments in this study were different, and direct comparison of the data collected from the two experiments is therefore necessarily not without difficulty, comparison of the general trends across the two experiments nevertheless provides an interesting opportunity to examine whether the type of prediction performance that is typically found in laboratory anticipation tasks (with simple, uncoupled responses) is indeed indicative of anticipation performance as it occurs in more natural settings (with actual, movement response requirements).

Figure 5 plots the skilled and novice data from Experiments 1 and 2 collected under the usual progressive temporal occlusion display condition. Consistently higher prediction scores are evident for the laboratory task (Experiment 1) compared to the field task (Experiment 2) suggesting, if the relative skill levels of the two different samples of participants and servers are similar, that the demands of the time constraints and the concurrent need to produce a movement response may make achievement of high prediction scores more difficult in the natural setting than in the laboratory. Consistently higher prediction scores are also apparent for the skilled participants in each experiment compared to their novice counterparts even though in each experiment in isolation, these differences failed to reach statistical significance. The most striking feature of Figure 5, however, is the remarkable parallelism of the plots for each skill group under each testing environment, indicating that both the laboratory and the field tests reveal the same picture with respect to the timing of information pick-up. For both skill groups, the consistent observation across both experimental settings is of information pick-up occurring in the $\mathrm{t} 3$-t 4 and $\mathrm{t} 4-\mathrm{t} 5$ time windows - as noted earlier, more fine-grained analyses within this $\sim 600 \mathrm{~ms}$ time period may be needed to show the difference in timing of information pick-up revealed in some other studies (cf. Abernethy \& Russell, 1987; Goulet et al., 1989). There is also evidence across both settings of a curious but consistent decrease in mean prediction accuracy from $\mathrm{t} 2-\mathrm{t} 3$, especially for the skilled players, suggesting that the provision of additional information in this 300 ms time window may be actually deceiving or misleading. This warrants further investigation, especially as it is not the first time such reductions in prediction accuracy have been noted in this period (cf. Farrow \& Abernethy, 2002). As the position of peak ball toss relative to the head of the server has been previously shown to be a biomechanical correlate of service direction (Hernandez \& Sicilia, 1998), it is conceivable that it is some element of the ball toss information available within this time window that is the root cause of this prediction accuracy loss. 


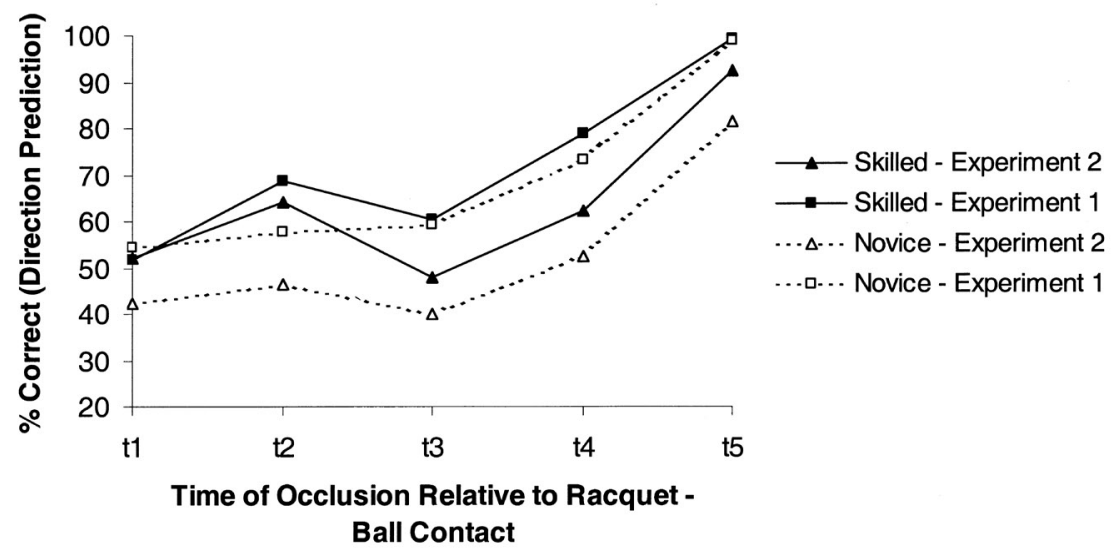

Figure 5-Prediction accuracy as a function of time of occlusion under the progressive temporal occlusion display condition for the skilled and novice participants in Experiment 1 (laboratory-based) and Experiment 2 (field-based).

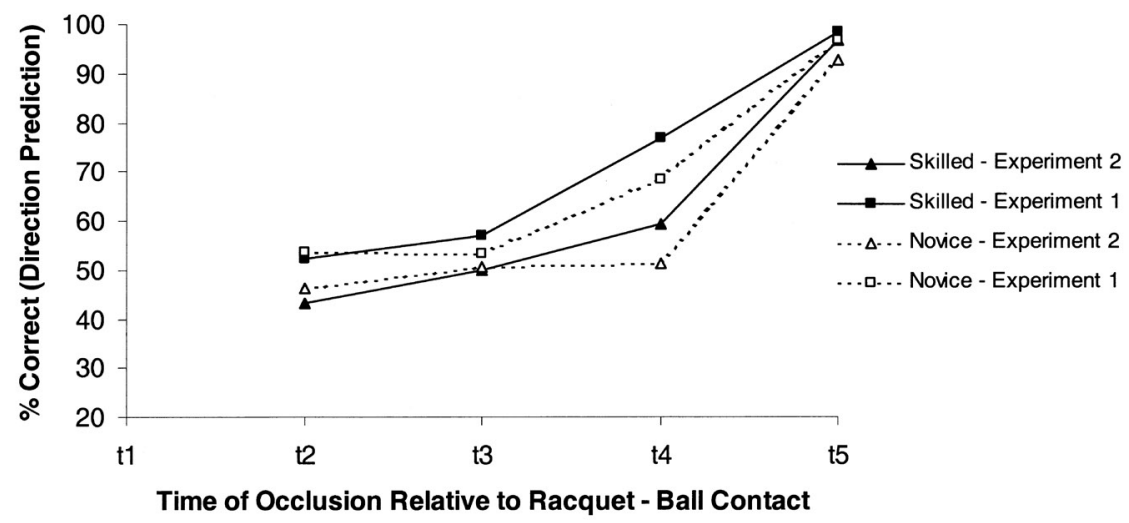

Figure 6-Predi

Figure 6 displays the prediction accuracy data collected from the moving window conditions in Experiments 1 and 2. Again, within the limitation that these are data derived from different participants and different servers, similar observations become apparent to those noted from the progressive occlusion conditions (Figure 5). Prediction scores on the laboratory task are consistently higher than those from the field task; the skilled consistently outperform the novice participants (at least at the 44 and 15 occlusions); and the general pattern of prediction improvement/information pick-up over time is similar across the two settings with both groups, improving in the $\mathrm{t} 3-\mathrm{t} 4$ and $\mathrm{t} 4-\mathrm{t} 5$ period (the only exception being the lack of improvement from $\mathrm{t} 3-\mathrm{t} 4$ for the novices in the field setting previously 
described). Interestingly, in the moving window display conditions, there is no evidence of the prediction performance decrements evident for $\mathrm{t} 2 \mathrm{-t} 3$ under the progressive display conditions suggesting that any deception effect that arises in $\mathrm{t} 2-\mathrm{t} 3$ is possibly a cumulative/carryover effect from events beginning in an earlier time period.

In summary, the bulk of the evidence collected in this study indicates that the conclusions that have been reached to date from laboratory studies using the progressive temporal occlusion paradigm are likely to be valid ones. Experiments 1 and 2 have provided evidence to indicate that the conclusions regarding information pick-up from the progressive temporal occlusion paradigm are ones that are replicable under conditions where viewing period duration is controlled and where the stimulus array, the time constraints, and the mode of responding are faithful to the natural task. Further diligent usage of the temporal occlusion paradigm through careful matching of key kinematic events in the movement pattern of interest with specific periods of visual occlusion has the potential to continue to provide further insights into the information sources used by performers of different skill levels.

\section{Acknowledgments}

Appreciation is expressed to the Australian Sports Commission, and in particular to the Australian Institute of Sport Tennis program, for the funding of Experiment 2. The authors are most grateful to Adam Gorman and Daniel Hornery for assistance with data collection and management in Experiment 1; Rob Bryant for technical expertise and assistance with the second experiment; and Chris Kachel, Graham Brimblecombe, and Mark McGrath (Australian Institute of Sport Tennis Program) and Geoff Masters (Queensland Academy of Sport Head Tennis Program) for the organization and provision of the players during the testing period.

\section{References}

Abernethy, B. (1990). Anticipation in squash: Differences in advance cue utilisation between expert and novice players. Journal of Sport Sciences, 8, 17-34. /aq/ This reference not found in text.//

Abernethy, B., Gill, D., Parks, S.L., \& Packer, S.T. (2001) Expertise and the perception of kinematic and situational probability information. Perception, 30, 233-252.

Abernethy, B., \& Russell, D.G. (1987). Expert-novice differences in an applied selective attention task. Journal of Sport Psychology, 9, 326-345.

Adam, J.J., \& Wilberg, R.B. (1992). Individual differences in visual information processing rate and the prediction of performance differences in team sports: A preliminary investigation. Journal of Sports Sciences, 10, 261-273.

Bennett, S., Ashford, D., \& Elliott, D. (2003). Intermittent vision and one-handed catching: The temporal limits of binocular and monocular integration. Motor Control, 7, 378-387.

Bennett, S., Elliott, D., Weeks, D.J., \& Keil, D. (2003). The effects of intermittent vision on prehension under binocular and monocular viewing. Motor Control, 7, 46-56.

Bootsma, R.J. (1989). Accuracy of perceptual processes subserving different perceptionaction systems. The Quarterly Journal of Experimental Psychology, 41A, 489-500.

Buckolz, E., Prapavesis, H., \& Fairs, J. (1988). Advance cues and their use in predicting tennis passing shots. Canadian Journal of Sport Science, 13, 20-30. 
Davids, K. (1988). Ecological validity in understanding sports performance: Some problems of definition. Quest, 40, 126-136.

Deary, I.J., \& Mitchell, H. (1989). Inspection time and high speed ball games. Perception, $18,789-792$.

Elliott, D., Chua, R., \& Pollock, B.J. (1994). The influence of intermittent vision on manual aiming. Acta Psychologica, 85, 1-13.

Farrow, D., \& Abernethy, B. (2002). Can anticipatory skills be learned through implicit video-based perceptual training? Journal of Sports Sciences, 20, 471-485.

Farrow, D., \& Abernethy, B. (2003). Do expertise and the degree of perception-action coupling affect natural anticipatory performance? Perception, 32, 1127-1139.

Goulet, C., Bard, C., \& Fleury, M. (1989). Expertise differences in preparing to return a tennis serve: A visual information processing approach. Journal of Sport and Exercise Psychology, 11, 382-398.

Helsen, W., \& Pauwels, J.M. (1988). The use of a simulator in evaluation and training of tactical skills in soccer. In T. Reilly, A. Lees, K. Davids, \& W.J. Murphey (Eds.), Science and football (pp. 493-497). London: E \& F.N. Spon.

Hernandez, F.M., \& Sicilia, A.O. (1998). Analysis of a professional tennis player to determine anticipatory pre-cues in the service. Journal of Human Movement Studies, 35, 219-231.

Jones, C.M., \& Miles, T.R. (1978). Use of advance cues in predicting the flight of a lawn tennis ball. Journal of Human Movement Studies, 4, 231-235.

Lyons, J., Fontaine, R., \& Elliott, D. (1997). I lost it in the lights: the effects of predictable and intermittent vision on unimanual catching. Journal of Motor Behavior, 29, 113-118.

Milgram, P. (1987). A spectacle-mounted liquid-crystal tachistoscope. Behavior Research Methods, Instruments \& Computers, 19, 449-456.

Paull, G., \& Glencross, D. (1997). Expert perception and decision making in baseball. International Journal of Sports Psychology, 28, 35-56.

Robertson, S., Collins, J., Elliott, D., \& Starkes, J. (1994). The influence of skill and intermittent vision on dynamic balance. Journal of Motor Behavior, 26, 333-339.

Sharp, R.H., \& Whiting, H.T.A., (1974). Exposure and occluded duration effects in a ballcatching task. Journal of Motor Behavior, 6, 139-147.

Starkes, J.L., Edwards, P., Dissanayake, P., \& Dunn, T. (1995). A new technology and field test of advance cue usage in volleyball. Research Quarterly for Exercise and Sport, $66,162-167$.

Whiting, H.T.A., \& Sharp, R.H. (1974). Visual occlusion factors in a discrete ball-catching skill. Journal of Motor Behavior, 6, 11-16.

Williams, A.M., Ward, P., Knowles, J.M., \& Smeeton, N.J. (2002). Anticipation skill in a real-world task: Measurement, training and transfer in tennis. Journal of Experimental Psychology: Applied, 8, 259-270.

Wright, D.L, Pleasants, F., \& Gomez-Meza, M. (1990). Use of advanced visual cue sources in volleyball. Journal of Sport and Exercise Psychology, 12, 406-414.

\footnotetext{
${ }^{3}$ As for Experiment 1, a preliminary four-way ANOVA including server as an additional factor was conducted and this revealed a main effect for server, $\mathrm{F}(1,14)=10.95, \mathrm{p}<.05$, but no simple interaction with the other factors of skill, $\mathrm{F}(1,14)=0.20, \mathrm{p}>.05$; display condition, $\mathrm{F}(1,14)=0.06$, $\mathrm{p}>$ .05 ; or occlusion level, $\mathrm{F}(3,42)=0.72, \mathrm{p}>.05$ nor any higher level interactions $(\mathrm{p}>.05)$. Again, one server was easier to predict than the other across all conditions but importantly, this did not interact with any other factors in the analysis, thereby permitting legitimate removal of the server factor from the main analysis.
} 
Copyright of Motor Control is the property of Human Kinetics Publishers, Inc.. The copyright in an individual article may be maintained by the author in certain cases. Content may not be copied or emailed to multiple sites or posted to a listserv without the copyright holder's express written permission. However, users may print, download, or email articles for individual use. 